\title{
Depressão em Adolescentes: Revisão da Literatura e o Lugar da Pesquisa Fenomenológica
}

\author{
Anna Karynne Melo \\ Adolfo Jesiel Siebra \\ Universidade de Fortaleza, CE, Brasil. \\ Universidade de Fortaleza, CE, Brasil. \\ Virginia Moreira \\ Universidade de Fortaleza, CE, Brasil.
}

\begin{abstract}
Resumo: Atualmente a depressão em adolescentes consiste em um fenômeno complexo e cada vez mais recorrente. Visando identificar e discutir os estudos que estão sendo produzidos sobre este tema, realizamos uma revisão de literatura com base em pesquisas indexadas nos bancos de dados SciELO; Portal de Periódicos da Capes; BDTD; APA; ScienceDirect, Redalyc; Lillacs e MedLine, mediante cruzamento das palavras-chave: depressão e adolescência; depressão em adolescentes; depressioninadolescentes;depresiónenadolescentes. Foramidentificados 247 textos, sendo que, após apreciação baseando-se nos critérios de elegibilidade, restaram 159 estudos. Num primeiro momento, essas pesquisas foram organizadas quantitativamente e, a seguir, por uma análise qualitativa. Os estudos evidenciaram cinco categorias temáticas: sintomas depressivos; fatores associados à variabilidade sintomatológica; eventos estressores e fatores de risco; comorbidades associadas à depressão em adolescentes e depressão e suicídio em adolescentes. Os eixos abordados demonstram que ainda existe muito a discorrer sobre essa forma de adoecimento, em virtude dos múltiplos contornos que a constituem. Destacamos a escassez de trabalhos pautados em uma perspectiva fenomenológica, apesar do gradativo crescimento nos últimos anos. Sugere-se a fomentação, bem como a construção de novos espaços de discussão, no intuito de auxiliar os profissionais que estão envolvidos com essa temática em sua praxis.
\end{abstract}

Palavras-chave: Depressão, Adolescentes, Revisão de Literatura, Fenomenologia.

\section{Depression in Adolescents: Review of the Literature and the Place of Phenomenological Research}

\begin{abstract}
Actually the depression in adolescents consists of a complex and increasingly recurring phenomenon. To identify and discuss the studies being produced on this topic conducted a literature review based on indexed searches on the banks of SciElo data; Journal Portal Capes; BDTD; APA; ScienceDirect; Redalyc; Lillacs and MedLine, by crossing the keywords: depressão $e$ adolescência; depression and adolescence; depressão em adolescentes; depression in adolescents; depression en adolescents. Identified 247 texts and after assessment based on the eligibility criteria, remaining 159 studies. At first these surveys were organizes quantitatively and then, for a qualitative analysis. The studies evidenced five thematic categories: depressive symptoms; factors associated with symptomatic variability; stressful events and risk factors; comorbidities associated with adolescent depression and depression and suicide in adolescents. The addressed axes show that there is still much to discuss this form of disease, because of multiple contours that constitute it. We highlight the shortage of guided work in a phenomenological perspective, despite the gradual growth in recent years. It is suggested to fostering and building new spaces for discussion, in order to help professionals who are involved with this theme in his praxis.
\end{abstract}

Keywords: Depression, Adolescents, Literature Review, Phenomenology. 


\title{
Depresión en Adolescentes: Revisión de la Literatura y el Lugar de la Investigación Fenomenológica
}

\begin{abstract}
Resumen: Actualmente la depresión en los adolescentes consiste en un fenómeno complejo y cada vez más recurrente. Identificar y analizar los estudios que se producen en este tema llevó a cabo una revisión de la literatura en base a búsquedas indexadas en las orillas de datos SciELO; Diario Portal Capes; BDTD; APA; ScienceDirect, Redalyc; Lillacs y MedLine, cruzando la depresión palabras clave y la adolescencia; depressão em adolescentes; depression in adolescentes; depresión en adolescentes. Se identificaron 247 textos, y después de una evaluación basada en los criterios de elegibilidad, permaneciendo 159 estudios. Al principio se organizaron estas encuestas cuantitativamente y luego, para un análisis cualitativo. Los estudios mostraron cinco categorías temáticas: síntomas depresivos; factores asociados con la variabilidad sintomática; eventos y factores de riesgo de estrés; comorbilidades asociadas con la depresión en adolescentes y la depresión y el suicidio en los adolescentes. Los ejes abordados muestran que todavía hay mucho que discutir esta forma de la enfermedad, debido a las múltiples contornos que lo constituyen. Destacamos la escasez de trabajo guiado en una perspectiva fenomenológica, a pesar del crecimiento gradual en los últimos años. Se sugiere el fomento y la creación de nuevos espacios para la discusión, con el fin de ayudar a los profesionales que están involucrados con este tema en su praxis.
\end{abstract}

Palabras clave: Depresión, Adolescentes, Revisión de Literatura, Fenomenología.

\section{Introdução}

A depressão consiste em um distúrbio que pode atingir o ser humano nos diferentes estágios da vida (Crivelatti, Durman, \& Hofstatter, 2006). Entretanto, a compreensão de que sujeitos antes da fase adulta também poderiam ser acometidos por esta enfermidade constitui-se em um campo recente de investigação, visto que até algumas décadas atrás, esse quadro clínico em crianças e adolescentes não era explorado.

Na década de 1970, os estudos associando depressão e infância/adolescência começaram a despertar um maior interesse. Suplicy (1993) assinala que dois fatores foram essenciais para essa mudança: o primeiro refere-se ao reconhecimento da comunidade científica do respectivo quadro clínico, fato ocorrido oficialmente em 1971, no $4^{\circ}$ Congresso da União Europeia de Psiquiatria Infantil; e o segundo motivo corresponde ao desenvolvimento das escalas de avaliação, ocasionando um aprimoramento nos diagnósticos sobre a depressão, tornando-os mais descritivos (Coutinho, 2001; Monteiro, \& Lage, 2007). Esse interesse culminou com a legitimação, em 1975, do diagnóstico da depressão em crianças e adolescentes por parte do Instituto Nacional de Saúde Mental dos EUA (NIMH) (Bahls, \& Bahls, 2002), o que contribuiu decisivamente para a enorme ascensão de trabalhos sobre a depressão nas décadas subsequentes.
Nos últimos anos, pesquisas como a de Souza et al. (2008) assinalam a presença cada vez mais significativa de adolescentes, em idade média de 16 anos, que apresentam uma sintomatologia depressiva, sendo esta atualmente considerada a doença mais frequente nesta fase (WHO, 2014). Esses dados preocupantes transformam a depressão, em especial na adolescência, em um problema de saúde pública, pois apresenta altas taxas de reincidência (Gladstone, Beardslee, \& O'Connor, 2011) e tem consequências que podem acompanhar a vida inteira do adolescente, de forma a debilitá-la (Avanci, Assis, \& Oliveira, 2008; Gladstone et al., 2011; Pelkonen, Marttunen, Kaprio, Hurrre, \&Aro, 2008; Souza et al., 2008).

Ante essas questões, entendemos a importância de estudos que possibilitem uma melhor compreensão sobre a depressão em adolescentes e suas diversas nuances. Neste artigo, realizamos uma revisão sistemática da literatura com o propósito de identificar e discutir as pesquisas que estão sendo produzidas no tocante a esse objeto de estudo.

\section{Método}

A revisão sistemática da literatura consiste em um método que visa a integração de informações simultâneas, a partir de procedimentos explícitos 
e sistemáticos, no intuito de identificar, analisar e avaliar os estudos que atendam aos critérios de elegibilidade estabelecidos. (Atallah, \& Castro, 1997; Higgins, \& Green, 2011; Munõz, Takayanagui, Santos, \& Sanchez-Sweatman, 2002; Rother, 2007). Ela utiliza como técnica para coleta de dados a consulta a fontes bibliográficas e eletrônicas (Gerhardt, \& Silveira, 2009). Tendo como parâmetro os modelos de Sampaio e Mancini (2007), bem como Gerhardt e Silveira (2009), a estratégia de busca seguida nesta pesquisa apresenta seis passos: definição da pergunta inicial; eleição dos descritores; identificação das bases de dados; definição dos critérios de exclusão e inclusão; coleta dos textos e análise dos estudos.

$\mathrm{O}$ questionamento que norteia este estudo e as demais etapas é: o que tem sido produzido na literatura científica, no campo da saúde, nacional e internacional e da psicopatologia sobre a depressão em adolescentes nos últimos 10 anos? Para tal pergunta, elencamos os seguintes descritores: depressão e adolescência; depressão em adolescentes; depression in adolescents; depresión en adolescentes. Optamos pela combinação de termos para obtermos uma busca mais focada no tema. A seguir, estabelecemos as bases de dados, levando em consideração a facilidade e a gratuidade do acesso: Scientific Eletronic Library On-line (SciELO); Coordenação e Aperfeiçoamento de Pessoal de Nível Superior (Portal de Periódicos da Capes); Biblioteca Digital Brasileira de Teses e Dissertações (BDTD); American Psychological Association (APA); ScienceDirect, Rede de Revistas Científicas de América Latina y el Caribe, España y Portugal (Redalyc); Literatura Latino-americana de Ciência da Saúde (Lillacs) e Medical Literature Analysis and Retrieval System On-line (MedLine).

Como passo seguinte, elegemos os critérios de inclusão e exclusão dos textos. Os de inclusão foram: apresentar como data de produção o período entre 2002 a 2012; adotar um conceito de adolescência; ser escritos em pelo menos um desses idiomas: português, inglês ou espanhol; apresentar a depressão em adolescentes entre as temáticas principais; ter como modalidade de produção científica: estudos empíricos, relatos de pesquisa, estudos de caso e revisão de literatura; estudos que foquem como amostra os adolescentes. Enquanto os critérios de exclusão foram: ser predominantemente teórico; ser um estudo que verifique a eficácia de alguma intervenção; ser uma pesquisa que tenha como objetivo a validação de escalas; não apresentar uma formatação, baseada na Associação Brasileira de Normas Técnicas (ABNT), ou na APA.

A coleta dos textos ocorreu durante todo o ano de 2013. Inicialmente, foram lidos o título e o resumo, porém, quando estes não deixavam claro se a pesquisa possuía todos os critérios de inclusão adotados, o texto integral era examinado. Primeiramente foram selecionados 247 textos, contudo, após uma segunda inspeção, foram excluídos 59 trabalhos por não explicitarem a natureza da pesquisa ou não apresentarem a normatização científica estipulada (por exemplo, resenhas, resumos, capítulos de livros, notícias).

A última parte do processo refere-se à análise dos estudos. Nessa etapa, outras produções também foram eliminadas $(n=29)$ em virtude de referência em duplicata e por apresentarem, enquanto modalidade de produção científica, a validação de escalas ou modelos de intervenção. Ao final desse procedimento restaram 159 artigos, que foram analisados.

\section{Resultados}

Foi realizada inicialmente uma descrição quantitativa dos dados, na qual os textos foram agrupados de acordo com: o número de pesquisas por ano; as revistas com maior frequência em publicações sobre esse tema; a quantidade de produções por base de dados e a natureza da pesquisa. Ademais, em um momento posterior, os 159 estudos selecionados foram examinados integralmente, sendo realizada uma descrição e análise qualitativa sobre os principais resultados.

\section{Descrição Quantitativa}

Ao examinarmos os 159 estudos remanescentes do processo, constatamos que cerca de $42 \%$ das produções são nacionais $(n=71)$, enquanto que $58 \%$ são internacionais $(n=88)$. Além disso, através da Tabela 1 foi possível identificar a disposição das pesquisas durante o período de 2002 a 2012.

A frequência anual dos estudos acerca da depressão em adolescentes exposta na Tabela 1 foi definida mediante o cruzamento das seguintes variáveis: número de pesquisas $\mathrm{X}$ tempo. Na primeira, utilizamos como referência números múltiplos de 5 , iniciando a mensuração a partir do 0 até o número 25, seguindo, portanto, a ordenação: 0-510-15-20-25. Em relação a segunda variável opta- 
mos por trabalhar com o intervalo de tempo anual, tomando como base o recorte temporal delineado no artigo, a começar pelo ano de 2002 até 2012, utilizando, portanto, a seguinte distribuição: 20022003-2004-2005-2006-2007-2008-2009-2010-20112012. Através dessa articulação, observamos que em 2002 foram publicados 6 artigos, enquanto que no ano seguinte (2003) foram apenas 4 . Entretanto, em 2004, a quantidade de publicação saltou para 9, chegando a 12 em 2005, 18 em 2006, 19 em 2007, 20 em 2008, 19 em 2009, 19 em 2010 e 20 em 2011. Já em 2013 constatamos uma redução no número de publicações, totalizando apenas 13 .

Diante do exposto, verificamos que, a partir do ano de 2003, houve um aumento gradativo de estudos envolvendo essa temática, passando de quatro para $18 \mathrm{em}$ apenas três anos. As revistas com o maior número de pesquisas foram: Journal of Affective Disorders $(\mathrm{n}=7)$; Journal of Abnormal Psychology $(\mathrm{n}=6)$; Ciência \& Saúde Coletiva $(\mathrm{n}=5)$.

Em relação à quantidade de pesquisas por base de dados, exposta na Tabela 2, os números encontrados demonstram que as fontes com maior quantidade de produções científicas são internacionais (APA e Science), seguidas pelo Portal da Capes, em terceiro lugar. No que se refere à natureza dos estudos, observamos uma predominância pelo relato de pesquisas
( $\mathrm{n}=140)$, seguido de revisões de literatura $(\mathrm{n}=17)$ e estudos de caso $(n=2)$.

A formulação da Tabela 2 contendo a quantidade de pesquisas por base de dados foi produzida a partir da relação estabelecida entre o número de pesquisas e a base de dados em que as mesmas estão indexadas. Tendo em vista a quantidade de produções selecionadas, elegemos os números múltiplos de 5 enquanto medidas de referência para representar os valores obtidos por cada banco de dados, iniciando da marca 0 até o número 35, ou seja, sistematizando a disposição em: 0-5-10-15-20-25-30-35. As bases de dados exibidas na Tabela 2 foram as mesmas utilizadas durante o processo de coleta de artigos, a saber: APA, Lillacs, Capes, BDTD, MedLine, Redalyc, SciELO e Science. Mediante esse cruzamento, constatamos a incidência de artigos em cada uma delas, verificando que, com exceção da APA com 30, a Science com 29 e a Capes com 25 produções, as bases de dados restantes transitam pela média de 15 artigos cada, sendo a menor quantidade verificada na Redalyc com apenas 11 estudos.

No tocante às revisões de literatura, houve uma maior quantidade de publicações em 2002, havendo variações nos anos seguintes entre uma a três revisões, contudo, em 2012, não foi encontrado nenhuma produção nessa modalidade. As revistas mais frequentes que apresentam esse tipo de estudo são: Psicologia

\section{Tabela 1}

Frequência anual dos estudos sobre depressão em adolescentes.

\section{Frequência Anual}

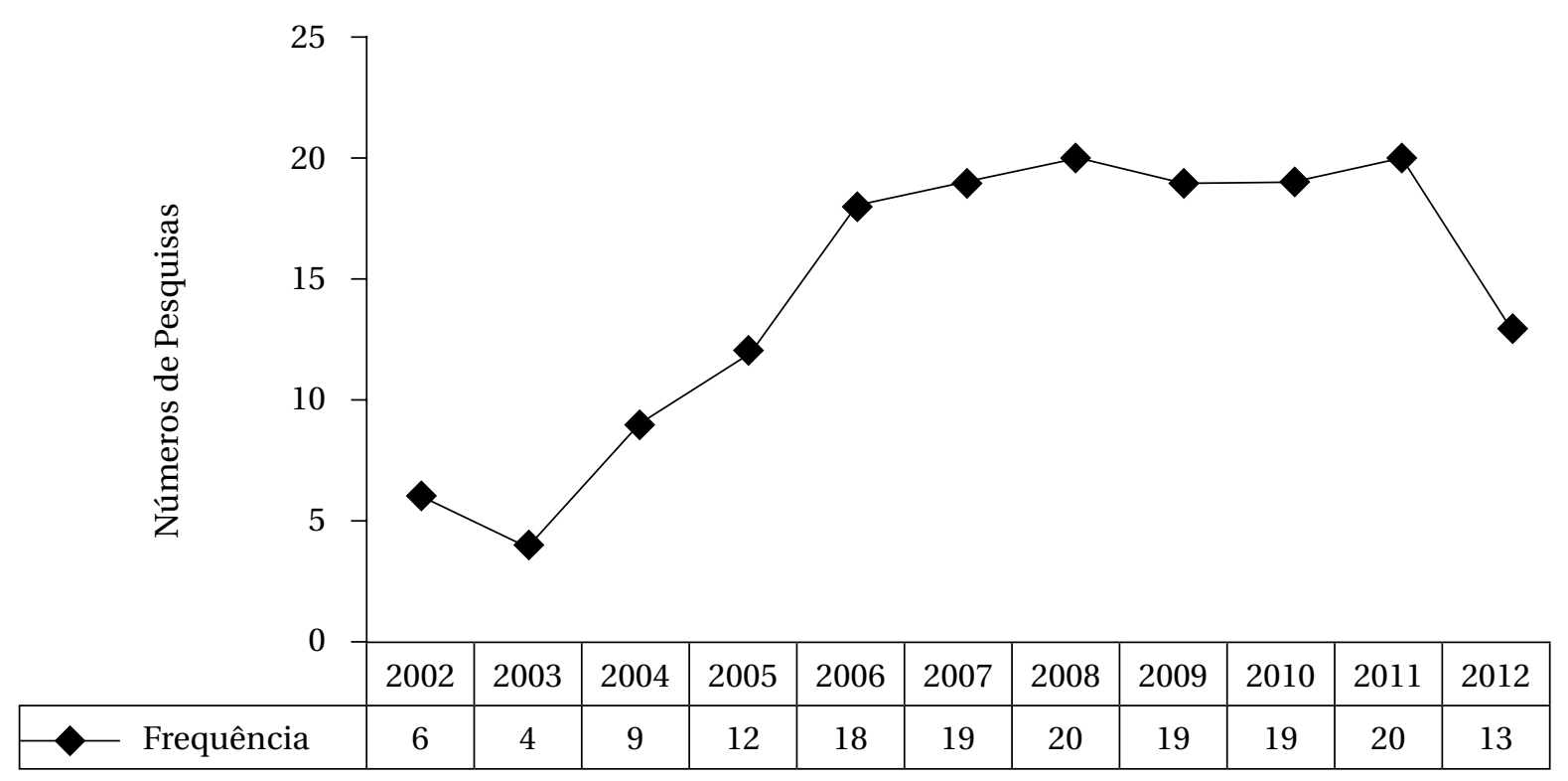


em Estudo, Revista de Psiquiatria Clínica e Jornal de Pediatria, tendo duas revisões cada.

\section{Descrição qualitativa}

Nesta etapa, os estudos foram agrupados de acordo com a semelhança de conteúdo. Nesse sentido, com base nas leituras das pesquisas, observamos que, de modo geral, os trabalhos elaborados abordaram com maior recorrência cinco grandes subtemas de investigação, organizados nas seguintes categorias temáticas: sintomas depressivos; fatores associados à variabilidade sintomatológica; eventos estressores e fatores de risco; comorbidades associadas à depressão em adolescentes e, por fim, depressão e suicídio em adolescentes.

\section{Os sintomas depressivos}

Bahls (2002) afirma que os sintomas depressivos de adolescentes e adultos apresentam quadros clínicos semelhantes. A autora associa os sintomas de depressão maior descrito no DSM-IV-TR (Associação Psiquiatrica Americana, 2002) aos existentes na depressão em adolescentes. Contudo, embora a autora realize essa aproximação, observa-se que nesse manual a depressão na adolescência não possui uma classificação específica, pois o que se constata são algumas observações em relação a esse quadro clínico. Como exemplo de tais retificações mencionamos: a substituição dos requisitos essenciais por irritabilidade, haja vista que "em crianças e adolescentes, pode-se desenvolver um humor irritável ou rabugento, em vez de humor triste e abatido" (Associação Psiquiatrica Americana, 2002, p. 349); a ressalva no tocante a relatividade sintomatológica devido à idade, visto que "os sintomas básicos para um Episódio Depressivo Maior são os mesmos para crianças e adolescentes, embora existam dados sugerindo que a predominância de sintomas característicos pode mudar com a idade" (p. 352). No DSM-V conserva-se a utilização de observações para fazer referência à possibilidade desse tipo de adoecimento em relação ao público adolescente, persistindo a tendência em não considerar a depressão nessa faixa etária uma categoria nosográfica específica (APA, 2013). A novidade em relação a esta edição fica a cargo da adesão de alguns especificadores como "com características mistas" e "com ansiedade" ao formular o diagnóstico de depressão. Além disso, percebemos uma maior preocupação na identificação cada vez mais precoce da depressão, visto que esta se apresenta como um primeiro indicativo de uma vulnerabilidade, trazendo o alerta da possibilidade do desenvolvimento de outros adoecimentos, considerados ainda mais graves, como a bipolaridade e a esquizofrenia.

\section{Tabela 2}

Quantidade de pesquisas por base de dados.

Número de Pesquisas X Banco de Dados

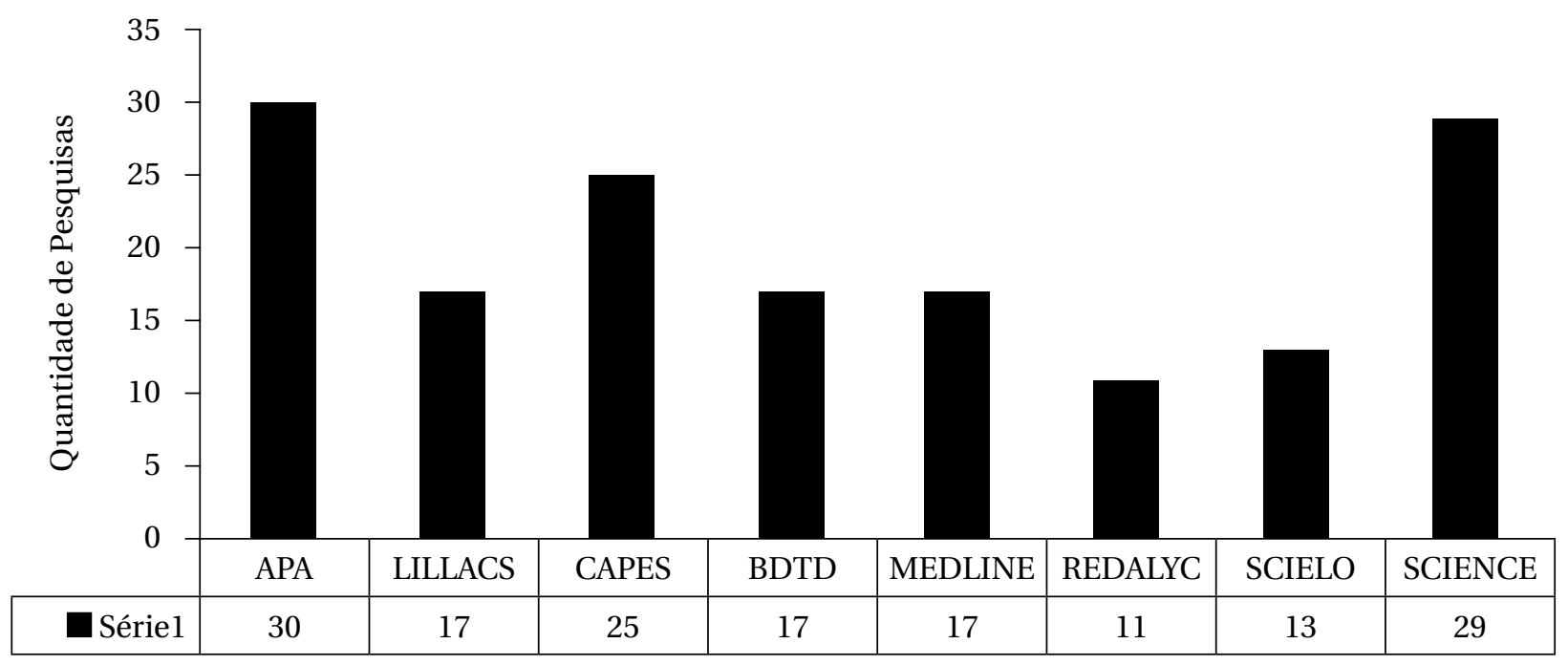


Dessa maneira, no intuito de possibilitar um diagnóstico mais preciso, Cunha, Buzaid, Watanabe e Romano (2005) elencam algumas características que são distintas nessa faixa etária, na tentativa de formular um quadro clínico mais específico e coeso. Entre eles, podemos citar a disforia com humor irritável, caracterizado por angústia, ansiedade, inquietação e agressividade, entendidos como dificuldade em lidar com seus sentimentos de baixa autoestima, desamparo e desapontamento consigo mesmo; a desesperança, com sensação de que as coisas não vão mudar; o abuso de álcool e outras drogas; o sentimento de inferioridade, inutilidade, descrevendo-se de modo negativo, autorrecriminação, solidão e hipocondria; a queda do desempenho escolar; a perda do interesse por coisas antes consideradas importantes; e a alteração do sono com frequente hipersonia.

Ao examinar esse grande número de sintomas, constatamos que, apesar de certo consenso, ainda são encontradas significativas discrepâncias na nosografia, atestando a dificuldade em formular um quadro clínico fidedigno e válido, reconhecido e legitimado pela maioria.

Fatores associados à variabilidade sintomatológica

Devido à falta de entendimento em relação aos sintomas e, consequentemente, aos diagnósticos, vários autores têm procurado identificar alguns fatores que possam estar relacionados a essa variabilidade sintomatológica. Lima (2004), por exemplo, defende que a idade é um fator preponderante, visto que " [...] quanto menor a criança, mais somáticos são os sintomas apresentados e mais a irritabilidade está presente" (p. 14). Rodríguez de Cossío e Granada Jiménez (2007) complementam que "la evaluación diagnóstica es más difícil a medida que disminuye la edad" (p. 271), pois as crianças pequenas não conseguem reconhecer e comunicar o que está lhe acontecendo.

Fu-l eWang (2008) verificaram a existência de alguns sintomas mais frequentes em crianças e adolescentes, como anedonia (72,4\%), humor depressivo $(72,4 \%)$, culpa excessiva $(62,1 \%)$, diminuição de concentração $(62,1 \%)$ e irritabilidade (58,6\%). Além disso, os autores observaram que os adolescentes apresentavam com maior intensidade certas manifestações depressivas, como humor depressivo, baixa autoestima e diminuição da concentração. Toros et al. (2004), realizando um estudo semelhante, ampliam essa lista enfatizando a tristeza, o vazio, a irritabilidade e os distúrbios somáticos.
Entretanto, pesquisas apuraram que as diferenças nas manifestações depressivas não se restringem apenas ao aspecto da idade. Jatobá e Bastos (2007), procurando identificar o predomínio de depressão e ansiedade em estudantes da cidade de Recife, PE, encontraram uma significativa relação do gênero feminino com sintomas depressivos de grande intensidade, considerando-os mais excessivos e frequentes nessa categoria. Rocha, Ribeiro, Pereira, Aveiro e Silva (2006), ao buscarem avaliar de que forma os sintomas depressivos estão distribuídos entre os alunos de uma Escola de Uberaba, MG, também encontraram uma maior incidência no sexo feminino, além de um gradativo aumento no número de casos acompanhando o progresso acadêmico. Analisando essa questão, Barros, Coutinho, Araújo e Castanha (2006) expõem que

[...] as meninas relatam mais sintomas subjetivos, como sentimentos de tristeza, vazio, tédio, raiva e ansiedade; costumam também ter mais preocupação com a popularidade, menos satisfação com a aparência, mais conscienciosidade e menos auto-estima, enquanto os meninos relatam mais sentimentos de desprezo, desafio e desdém, e apresentam problemas de conduta, como falta às aulas, fugas de casa, violência física, roubos e abuso de substâncias psicoativas (p. 20).

Stein et al. (2010) também assinalam que o meio no qual o sujeito se constitui afeta diretamente a forma e a intensidade como ele expressa seus sintomas. Melo e Moreira (2008) apontam a relação entre patologia e cultura. Ao analisar o prontuário de 20 adolescentes, na faixa etária de 15 a 18 anos, as autoras verificaram que as categorias encontradas são relacionadas diretamente com o contexto no qual os adolescentes estão inseridos, concluindo assim que "a experiência da depressão entre eles é constituída mutuamente com a cultura, sendo composta por contornos culturais, sociais e econômicos nos quais vivem os adolescentes" (p. 61).

Compreendemos que a percepção de mundo dos adolescentes tem relação direta com a maneira de exteriorizar esses sentimentos, conforme defendem Coutinho e Ramos (2008) quando assinalam que a presença de estados depressivos em várias culturas reafirma o caráter universal da depressão, considerando-a assim uma doença e não apenas um fenômeno social específico de uma sociedade. 
As diferentes manifestações sintomatológicas explanadas demonstram como essa enfermidade se constitui de maneira singular na adolescência, sendo importante considerar outros elementos que possam repercutir no desenvolvimento desta doença. Além disso, é preciso ficar atento para a possibilidade de um mesmo fator conter outras significações.

\section{Eventos estressores e fatores de risco}

A adolescência caracteriza-se por ser um período peculiar em virtude das várias transformações e exigências que ocorrem nessa época, pois é um momento em que o adolescente se depara com diversas situações que podem contribuir para a flutuação de humor e alterações significativas no comportamento (Ballone, \& Moura, 2008). Essas intensas mudanças a caracterizam como uma fase de reorganização, tornando-a extremamente propensa ao desenvolvimento de alguns distúrbios, estando a depressão entre os principais (Souza et al., 2008).

Segundo Poletto, Koller e Dell'Aglio (2009), a percepção que o sujeito tem de determinada situação influenciará a maneira como ele vai lidar com tais circunstâncias. Os autores expõem que a compreensão de evento estressor/fator de risco dependerá de como o sujeito se constitui, assim como o contexto no qual ele está inserido. Horwitz, Hill e King (2011) complementam alertando que as formas de enfrentamento ao lidar com eventos estressores podem indicar o desenvolvimento de depressão e ideação suicida. Em sua pesquisa, eles verificaram que retiradas comportamentais (por exemplo, retirada de comportamento) assim como autorresponsibilização (por exemplo, autoculpa) podem favorecer a emergência desses fenômenos.

Como todo indivíduo em sociedade, várias dimensões perpassam e atravessam esse sujeito, afetando-o de maneira singular. Logo, considerar uma situação estressora ou não dependerá diretamente do indivíduo, refletindo, assim, em uma variedade de possibilidades. Por isso, embora não se reconheça uma unanimidade na classificação de alguns fatores, no decorrer da pesquisa em questão, percebemos certo consenso entre os autores na classificação de determinadas variáveis como fatores de risco e/ou proteção, a saber: a vulnerabilidade social, o contexto familiar, suporte social, contexto social e comorbidades.

Quanto à vulnerabilidade social, Barros et al. (2006), com o propósito de verificar as representações sociais de adolescentes no cenário do ensino médio, realizaram um estudo comparativo entre duas escolas de João Pessoa, PB, sendo uma pública e a outra particular. Participaram da pesquisa 60 alunos, com faixa etária média de 16 anos e que apresentavam uma sintomatologia depressiva. Em seus resultados, os autores constataram que há uma divergência entre os adolescentes em relação à concepção etiológica da depressão, pois, ao serem questionados sobre a origem desse distúrbio, os estudantes da escola pública evidenciaram os aspectos psicossociais, exemplificadas por situações de desfavorecimento social e econômico, enquanto que os adolescentes da escola particular destacaram mais as dimensões afetivas e psicológicas.

Diante desse cenário, estudos (Bós, \& Bós, 2005; Gazalle, Lima, Tavares, \& Hallal, 2004; Kubik, Lytle, Birnbaum, Murray, \& Perry, 2003; Souza et al., 2008; Zinn-Souza et al., 2008) comprovam que a prevalência de depressão apresenta-se maior em indivíduos de classes sociais mais baixas, pois "nesses contextos, com muita frequência, além de privações típicas trazidas pela baixa renda, coexiste o desemprego, as uniões transitórias entre casais, a violência e o alcoolismo, confluindo para o aparecimento da depressão nessa fase da vida" (Máximo, 2010, p. 67).

Em relação à temática família, vários trabalhos têm comprovado o elo entre depressão e o contexto familiar. Na pesquisa de Avanci et al. (2008), por exemplo, realizada na cidade do Rio de Janeiro, com uma amostragem de 1.923 alunos entre 11 a 19 anos, os autores constataram que determinados adolescentes que manifestavam sintomas depressivos percebiam sua estrutura familiar como deficiente,

[...] composta por padrasto/madrasta ou sem figuras parentais; relacionamento regular/ ruim com pais e irmãos; ausência ou pouca supervisão familiar; fraco apoio emocional e baixa interação positiva. A ocorrência de eventos estressantes na família também associam-se à expressão da depressão, entre eles problemas financeiros, de saúde, com o uso de álcool e drogas, separação dos pais, novo casamento dos pais, prisão/indiciamento de um familiar, severa violência física cometida pelo pai e pela mãe contra o adolescente, testemunho dessa forma de violência entre os pais, vitimização de violência entre irmãos, violência psicológica vivida em níveis elevados e vitimização da violência sexual perpetrada pelos pais contra o adolescente (p. 2.339). 
Santana (2008) afirma que o contexto familiar é fundamental para o desenvolvimento do adolescente, pois possibilita um ambiente de proteção e tranquilidade contra as adversidades diárias, percebidas de forma mais intensa nessa etapa do desenvolvimento humano. Herman, Ostrander e Tucker (2007) complementam ressaltando como o vínculo entre pais e filhos é estruturante para a personalidade do adolescente. Contudo, a origem da depressão não pode ser simplificada em um pensamento linear, resultando em uma hipótese de causa e efeito, tendo em vista que para o surgimento dessa enfermidade, outros fatores também estão implicados. Ainda no contexto familiar, pesquisas têm sido feitas no intuito de validar as consequências das possíveis práticas parentais sobre os seus descendentes. De acordo com Palos, Ocampo, Casarín, Ochoa e Rivera (2012), os pais são os agentes primordiais da socialização e muitas das características emocionais, cognitivas e comportamentais dos filhos estão relacionadas às formas específicas de criação adotadas por eles. Na pesquisa realizada no México, com o propósito de examinar a relação práticas parentais com a sintomatologia depressiva dos filhos adolescentes, observou-se que adolescentes que consideravam ser controlados e percebiam uma educação impositiva, apresentavam frequência e intensidade sintomatológica maior em comparação aos que afirmavam que seus pais possuíam um canal mais aberto de comunicação que permitia o desenvolvimento de autonomia por parte dos adolescentes.

Araújo (2003) alerta que adolescentes com manifestações depressivas apresentam uma compreensão distorcida sobre os comportamentos dos pais, encarando-os como disfuncionais. Além disso, o autor relata que

[...] as investigações mostram, de forma consistente, que relações caracterizadas por insegurança, pouca proximidade emocional e estilos educativos parentais marcados por falta de apoio, interesse e carinho, se associam a perturbações psicopatológicas na idade adulta. Na sua maioria, os estudos apontam um efeito maior por parte das relações estabelecidas com a mãe, por contraste àquelas com o pai, no mesmo período de tempo (p. 217).
Champion et al. (2009) destacam que a dificuldade nas relações pode ser influenciada por um histórico de depressão materna. Na pesquisa efetuada por esses autores, constatou-se que filhos de mães possuidoras dessas características apresentam maior propensão ao quadro depressivo, assim como comportamentos estressantes. Reeb e Conger (2009), assim como Pawlby, Hay, Sharp, Waters e O'Keane (2009), ampliam essa compreensão, por meio de seus estudos, verificando que a sintomatologia encontrada nos pais apresenta expressiva ligação com os posteriores sintomas depressivos dos filhos adolescentes. Aliado a isso, Morris, Ciesla e Garber (2010) ressaltam que, devido a esse convívio com graus diferentes de depressão, o adolescente adquire uma maior vulnerabilidade a eventos estressores, sendo mais propenso aos sintomas depressivos (Bouma, Ormel, Verhulst, \& Oldehinkel, 2008).

Esse resultado é consoante aos encontrados em algumas pesquisas (Aragão, Coutinho, Araújo, \& Castanha, 2009; Baptista, \& Oliveira, 2004; Santana, 2008), nas quais os autores também reconhecem a essencial participação do meio, não só do familiar, mas também do social, na construção e desenvolvimento do adolescente. Stice, Ragan e Randall (2004) sublinham que a percepção dos apoios sociais possui uma forte influência na incidência de sintomas depressivos, pois existe uma significativa relação inversamente proporcional com depressão e suporte social. Ademais, eles observaram que o suporte social mais significativo no início da adolescência refere-se ao contexto familiar, enquanto que nos anos posteriores esse apoio é delegado aos pares.

Esse pensamento relaciona-se ao pesquisado por Schneider e Ramires (2007) que destacam, também, a relevância dessa rede de apoio mais próxima. Eles colocam que, à medida que os adolescentes crescem, as relações fraternas e de amizade adquirem um peso maior, pois o desprendimento gradual dos pais contribui para que o sujeito busque outras redes de apoio, principalmente entre seus pares, considerados nesse momento como iguais.

Wisdom e Agnor (2007) enfatizam que os pares, além da importância citada, também possuem uma relevante influência sobre o adolescente na decisão de procurar ajuda. Para os autores, a construção de um ambiente favorável possibilita o adolescente sentir-se seguro o suficiente para decidir e admitir que precisa de cuidado especializado, isso ocorre devido ao fato de o transtorno depressivo ainda ser considerado um estigma, principalmente para os seus portadores (Calear, Grifftihs, \& Chritensen, 2011). 
Comorbidades associadas à depressão em adolescentes

Outro ponto de grande destaque na literatura específica refere-se à alta taxa de comorbidade existente no adolescente que apresenta um quadro de depressão. Essa perspectiva tem como marco principal o CID-10 (OMS, 1993). Esse manual, assim como o DSM-IV-TR (Associação Psiquiátrica Americana, 2002), não considera a depressão na adolescência como uma categoria nosográfica própria. Contudo, a ressalva encontrada no DSM-IV (Associação Psiquiátrica Americana, 2002) e no DSM-V (APA, 2013) evidenciando algumas particularidades em relação a esse quadro clínico na adolescência não é observada no CID-10, sendo entendido como um quadro uniforme para todos. De acordo com Monteiro e Lage (2007), em relação ao CID-10,

[...] existem 36 subdivisões e a referência à manifestação do humor depressivo não está restrita somente aos transtornos de humor, mas em outros transtornos psiquiátricos, como, por exemplo: os fóbico-ansiosos, ansiedade generalizada, obsessivo-compulsivo e os transtornos depressivos de conduta (p. 259).

Neste manual, a menção feita para crianças e adolescentes referente à depressão pode ser encontrada apenas na categoria F92 (Transtorno Misto de Conduta e Emoções), que consiste na combinação entre F91 (Transtorno de Conduta) com Transtorno Depressivo (F30-39) (OMS, 1993). Hale, Van der Valk, Engels e Meeus (2005) destacam que a agressividade apresenta forte associação com a percepção dos adolescentes em relação ao nível de rejeição parental, e a depressão seria utilizada como um instrumento de mediação entre esses dois fatores. Em outros exemplos, pesquisas ratificam a prevalência de comorbidades, principalmente relacionado ao transtorno de ansiedade (McCauley, Katon, Russo, Richardson, \& Lozano, 2007; Silva Junior, 2010).

Constatamos, também, na literatura, uma frequente associação entre depressão e o uso de substâncias psicoativas. Andrade e Argimon (2008), por meio de uma revisão sistemática em base de dados computadorizadas no período de 2000 a 2005, verificaram a forte prevalência do uso de cannabis $^{1}$ na inci- dência de transtornos depressivos. Em seu levantamento, os autores perceberam a significativa utilização desse recurso como forma de enfrentamento do cotidiano por partes dos sujeitos que apresentavam esse quadro clínico. Além disso, notou-se que a precocidade no uso de cannabis tem expressiva relação com os sintomas depressivos.

Com a intenção de analisar o uso da maconha e o emprego dessa substância como auxílio para as adversidades do cotidiano e servindo como suporte para habilidades sociais, Wagner, Silva, Zanettelo e Oliveira (2010) realizaram um estudo comparativo em um grupo de 30 adolescentes, sendo 15 usuários de maconha e 15 não usuários de maconha. Por meio dele, foi possível examinar que o grupo contendo usuários apresentaram um desempenho mais deficiente no aspecto referente ao autocontrole da agressividade em situações agressivas. Segundo os autores, isso demonstra uma "maior inabilidade a lidar com sentimentos e reações de agressividade, gerados em situações sociais que vivenciam" (p. 263). Esses dados são condizentes com os encontrados em pesquisa como Campos (2010), que verifica a baixa frequência de habilidades sociais em adolescentes com indicadores de depressão.

Essa relação não se restringe ao uso de substâncias ilícitas (Clarke, \& Harvey, 2012), Zinn-Souza et al. (2008), buscando observar a associação entre determinados fatores com os distúrbios depressivos, verificaram que o consumo de bebidas alcoólicas está entre os principais. Os autores reconhecem que o contato prematuro com a bebida alcoólica favorece esse comportamento de risco. Teixeira, Stefanini, Martins e Cruz (2011) alertam, também, que o consumo significativo dessa substância compromete o rendimento acadêmico, ou, como defende Bossarte e Swahn (2011), aumenta a probabilidade de tentativas de suicídio.

Depressão e suicídio em adolescentes

Trata-se de uma das questões mais delicadas quando se aborda adolescentes com quadro clínico depressivo, pois o suicídio consiste na terceira maior causa de morte entre eles (WHO, 2014). No Brasil, segundo o Mapa da Violência 2012, verifica-se que os números de suicídios de maneira geral, dentro da faixa etária apresentada (>1 a 19 anos), não são

\footnotetext{
1 "Droga de efeito entorpecente preparada com os ramos, folhas e flores do cânhamo, cortados e secos, ger. curtidos em substâncias como o mel, conhaque etc., consumida como o tabaco, e cujo componente ativo é o tetraidrocanabinol"
} 
significativos. O documento aponta que, no cenário internacional, o Estado brasileiro ocupa sexagésimo lugar, ressaltando a posição confortável diante desse fenômeno. No entanto, também verificou um gradativo aumento nesses índices (2000-2010), demonstrando que estudos são necessários para averiguar essa tendência, pois, em algumas cidades e estados, os números são alarmantes. É interessante destacar que os episódios de suicídio na pesquisa só emergem no início da pré-adolescência, ou seja, por volta dos dez anos de idade, apresentando uma curva acentuada a partir de então, chegando ao seu ápice, em torno dos 18 e 19 anos (Waiselfisz, 2012).

Werlang, Borges e Fensterseifer (2005), no intuito de contribuir para que medidas preventivas em relação ao comportamento suicida em adolescentes sejam tomadas mais precocemente, buscaram, por meio de uma pesquisa com 526 adolescentes com idade entre os 15 e 19 anos, identificar quais os fatores de risco e proteção associados a essa atitude. Em seus achados, verificaram que dentre os fatores de risco relacionados ao suicídio estão: a ideação suicida; a organização familiar; o falecimento de alguns dos pais; a doença física debilitante; o conhecer alguém que tentou suicídio; e não ter feito qualquer acompanhamento psicológico. Em relação aos fatores de proteção, foi elencado a boa relação com os membros da família e o trabalho. No que tange a este último ponto, resultados similares foram encontrados nos estudos de Benincasa e Rezende (2006) que, nos dados obtidos em sua pesquisa, revelaram que, entre esses fatores de proteção, as categorias mais citadas foram relacionadas com "família próxima" e "alguém confiável pra se abrir".

Os estudos de Santos, Lovis, Legacy e Abelha (2009), bem como os de García, Montoya, Loyo, López e Gaitan (2011), ressaltam que a depressão apresenta-se como fator preditivo para o suicídio, não podendo ser "naturalizado" em virtude da fase. Além disso, Borges, Werlang e Copatti (2008) constataram que essa associação não é exclusiva de adolescentes depressivos graves. Eles verificaram que, a partir de uma depressão leve, o indivíduo possui a probabilidade de 1/12 de desenvolver ideação suicida. Bella, Fernández e Willington (2010) complementam mostrando que as tentativas prévias também são fatores essenciais para prever comportamentos suicidas em adolescentes.

\section{Análise da revisão sistemática da literatura e o lugar da pesquisa fenomenológica}

A literatura revelou que ainda existe certa dificuldade em construir um quadro nosográfico concreto para o diagnóstico da depressão em adolescentes, visto que a partir das pesquisas apresentadas verificou-se uma quantidade expressiva de possibilidades que indicam sua presença. Além disso, observamos que os aspectos no tocante a idade e o gênero devem ser levados em consideração ao iniciar o processo de compreensão desse fenômeno altamente complexo.

Os resultados obtidos contribuíram para constatarmos que alguns fatores potencializam o desenvolvimento da depressão em adolescentes, sendo importante garantir a prevenção, ou amenização desses aspectos, no intuito de evitar o agravamento dessa enfermidade que ocasiona repercussões extremamente danosas ao sujeito como o suicídio, umas das principais causas de morte nessa faixa etária. Ademais, ressaltamos a primazia da depressão na adolescência, não podendo ser considerada apenas como um efeito secundário ou que exista somente em comorbidade com outras doenças.

A revisão da literatura também anunciou alguns pontos que ainda necessitam de um maior aprofundamento, como a presença de estudos longitudinais no contexto brasileiro para acompanhar o desenvolvimento gradual da depressão em adolescentes, bem como a elaboração de pesquisas que busquem compreender a experiência depressiva de adolescentes.

Neste nosso estudo, ao identificarmos e analisarmos os resultados obtidos, evidenciamos a carência de estudos fenomenológicos sobre a adolescência e depressão. Apesar de não ter sido nosso objetivo inicial discutir este ponto, como estudiosos desta perspectiva teórica este fato nos chamou a atenção. Santiago e Holanda (2013) em seu estudo também encontraram um número reduzido de artigos sobre o tema da depressão na perspectiva fenomenológica. Embora o levantamento realizado por esses autores não tenha feito uma distinção em relação à idade, ao examinarmos as pesquisas selecionadas por estes autores que trabalhavam com a adolescência diretamente, verificamos que a quantidade de artigos diminui consideravelmente, passando de 21 para apenas um.

Todavia, em suas análises, os autores observaram que, no âmbito brasileiro, houve um crescimento progressivo de pesquisas fundamentadas por essa pers- 
pectiva teórica, principalmente nos últimos 15 anos. Uma dessas pesquisas, já citada, foi realizada numa clínica escola de Psicologia com adolescentes com queixas depressivas e o resultado foi que a condição socioeconômica, a relação familiar, a experiência da religião, o relacionamento afetivo, a percepção de si, a experiência com as drogas, a busca do findar o sofrimento e a dificuldade de interação social são aspectos constitutivos da experiência vivida da depressão destes adolescentes (Melo, \& Moreira, 2008).

Tal cenário reflete o aumento gradativo na adesão a esse referencial, devido à possibilidade que este oferece de realizar uma compreensão diferente do fenômeno em relação ao que tradicionalmente se utiliza - perspectiva biologicista e classificatória, contribuindo assim para a ampliação do entendimento desse adoecimento tão complexo e cada vez mais recorrente, que é a depressão. Esses achados demonstram que, embora busque-se elaborar construções teóricas que possam ser utilizados como parâmetro na investigação do processo de adoecimento, torna-se fundamental reconhecer que nenhuma teoria consegue conceber $o$ homem exaustivamente, em toda a sua realidade.

$O$ adolescente transita por um período de oscilações intensas e um dos meios para compreender de que forma essa experiência da depressão se constitui, é oportunizar um espaço onde ele possa descrever o seu mundo e seus significados, saindo assim de uma atitude natural pautada em realidades objetivas para compreender as condições de possibilidade dessa experiência (Moreira, 2009). Por este motivo, destacamos uma compreensão que, tomando como base a psicopatologia fenomenológica numa leitura de Arthur Tatossian, assinala a experiência como fenômeno e que somente a partir da experiência seja possível "apreender o modo de ser global do sujeito, as significações dos comportamentos e dos vividos" (Bloc, \& Moreira, 2013, p. 33).

Para a psicopatologia fenomenológica, torna-se necessário resgatar a ideia de pathos em toda a sua amplitude e como condição humana, já que, atualmente, ela tem sido reduzida ao sentido patológico. Vemos essa redução, no caso da adolescência, quando esta é entendida como um processo universal e com características patológicas. Entendemos que a fronteira entre o normal e o patológico, além de ser extremamente tênue, corresponde a uma construção social sustentada por valores e ideologias que tem tratado o sofrimento psíquico, na maioria das vezes, como uma doença (Moreira, \& Sloan, 2002). Para Martins (1999), é pela condição páthica do homem que este constrói uma maneira singular de estar-no-mundo, sendo o adoecimento apenas uma de suas possibilidades. Assim, ratifica-se que a constituição do adolescente não ocorre de forma alheia ao contexto que vive, pois ele se constitui com e no mundo, não sendo possível compreender esse sujeito restringindo o olhar aos seus aspectos individuais.

Segundo Moreira (2009), ao pensar esse sujeito enraizado no mundo, a compreensão de saúde deixa de ser entendida como algo estagnado a favor da ideia de um processo, "no qual o organismo se atualiza em conjunto com o mundo, transformando-se e atribuindo-lhe significado, na medida em que o ser humano se transforma" (p. 96). Nesse entendimento do entrelaçamento homem e mundo, para a autora, em seus estudos transculturais sobre a depressão, esta deve ser investigada como uma experiência cultural, pois emerge não só de processos psicológicos e estruturais, mas também, de processos políticos e sociais (Moreira, 2007).

\section{Considerações finais}

A depressão, apesar de ser um adoecimento bastante discutido e estudado, é um fenômeno ascendente da sociedade contemporânea, principalmente entre os adolescentes, sendo ainda objeto de grandes pesquisas em vários níveis. Nesse sentido, o intuito dessa revisão foi contribuir, através da explanação de pesquisas referente a essa temática, para que a compreensão sobre essa doença e suas repercussões nesses indivíduos seja ainda mais integral, exibindo quais são os conteúdos mais abordados e os que ainda carecem de um aprofundamento. Tal atitude procura desnaturalizar o fenômeno da depressão como algo já acabado, saturado em todos os seus aspectos.

Em nossos achados, percebemos que os modelos nosográficos atuais ainda se apresentam como insuficientes para contemplar a heterogeneidade de manifestações existentes nesse quadro clínico, devido à diversidade de fenômenos que os adolescentes expressam ao serem acometidos pela depressão, dificultando a elaboração de um quadro teórico que possa ser utilizado como subsídio pelos profissionais que atuam com esse público.

Para isso, ressaltamos a necessidade de considerar a singularidade que o processo depressivo apresenta, evidenciando aspectos como idade e gênero, 
como fundamentais para a compreensão desse adoecimento, bem como a significação que certos eventos possuem para os adolescentes, não podendo pressupor algo como prejudicial ou benéfico, sem antes compreender as vivências destes sujeitos e o sentido dado por eles a essas experiências. Isso não corresponde a um abandono do que já foi produzido, pelo contrário, a partir das pesquisas encontradas, constatamos como certos fatores requerem uma atenção maior, tendo em vista que podem prevenir ou agravar o desenvolvimento desta enfermidade, como o campo familiar e o social, por exemplo.

A depressão, antes considerada como um efeito secundário de outras enfermidades, atualmente, postula uma autonomia diante delas, demonstrando que, por si só, acarreta danos graves à vida do adolescente e que não necessariamente teria que estar atrelada a alguma comorbidade para ser considerada nociva ou prejudicial ao sujeito. Ademais, verificamos que a depressão apresenta uma relação muita próxima com o suicídio, temática tão delicada nessa faixa etária, tendo em vista que refere-se a uma das principais causas de morte nesse período.

A depressão na adolescência é um problema a ser considerado por diversos saberes como a Psicologia, a Psiquiatria, a Psicopatologia e a Saúde Mental, dentre outros, e entendemos que há a necessidade de uma postura reflexiva crítica, na qual o profissional pode analisar criticamente os múltiplos contornos que constituem a experiência vivida da depressão. Pesquisas fenomenológicas, ainda muito escassas, contribuiriam para a compreensão da depressão e do sujeito adolescente no seu mundo vivido, com implicações para a prática de cuidado com esse público.

\section{Referências}

American Psychiatric Association - APA (2013). Diagnostic and statistical manual of mental disorders (DSM-V) (5A ed.). Arlington, VA: American Psychiatric Association.

Andrade, T. M. R., \& Argimon, I. I. L. (2008). Sintomas depressivos e uso de Cannabis em adolescentes. Psicologia em Estudo, 13(3), 567-573. https://doi.org/10.1590/S1413-73722008000300018

Aragão, T. A., Coutinho, M. P. L., Araújo, L. F., \& Castanha, A. R. (2009). Uma perspectiva psicossocial da sintomatologia depressiva na adolescência. Ciência \& Saúde Coletiva, 14(2), 395-405. https://doi.org/10.1590/S1413-81232009000200009

Araújo, A. F. (2003) Percepção dos estilos educativos parentais e ajustamento psicológico do adulto: comparação entre indivíduos com e sem perturbações depressivas. Paidéia (Ribeirão Preto), 12(24), 215-227. https://doi.org/10.1590/S0103-863X2002000300010

Associação Psiquiátrica Americana (2002). Manual diagnóstico e estatístico de transtornos mentais (DSM IV-TR) (4a ed.). Porto Alegre, RS: Artmed.

Atallah, N. A., \& Castro, A. A. (1997). Revisões sistemáticas da literatura e metanálise: a melhor forma de evidência para tomada de decisão em saúde e a maneira mais rápida de atualização terapêutica. Diagnóstico \& Tratamento, 2(2), 12-15.

Avanci, J. Q., Assis, S. G., \& Oliveira, R. V. C. (2008). Sintomas depressivos na adolescência: estudo sobre fatores psicossociais em amostra de escolares de um município do Rio de Janeiro, Brasil. Cadernos de Saúde Pública, 24(10), 2334-2346. https://doi.org/10.1590/S0102-311X2008001000014

Bahls, S. C. (2002). Aspectos clínicos da depressão em crianças e adolescentes. Jornal de Pediatria, 78(5), 359-366. https://doi.org/10.1590/S0021-75572002000500004

Bahls, S. C., \& Bahls, F. R. C. (2002). Depressão na adolescência: características clínicas. Interação em Psicologia, 6(1), 49-57.

Ballone G. J., \& Moura E. C. (2008). Depressão na adolescência. Recuperado de http://www.psiqweb.med.br/site/?area $=\mathrm{NO} /$ LerNoticia\&idN oticia $=129$

Baptista, M. N., \& Oliveira, A. A. (2004). Sintomatologia de depressão e suporte familiar em adolescentes: um estudo de correlação. Revista Brasileira de Crescimento e Desenvolvimento Humano, 14(3), 58-67. https:// doi.org/10.7322/jhgd.40168 
Barros, A. P. R., Coutinho, M. P. L., Araújo, L. F., \& Castanha, A. R. (2006). As representações sociais da depressão em adolescentes no contexto do ensino médio. Estudos de Psicologia (Campinas), 23(1), 19-28. https://doi.org/10.1590/S0103-166X2006000100003

Bella, M. E., Fernández, R., \&Willington, J. M. (2010). Intento de suicidio en niños y adolescentes: depresión y trastorno de conducta disocial como patologías mas frecuentes. Archivos Argentinos de Pediatria, 108(2), $124-129$. Recuperado de http://www.scielo.org.ar/scielo.php?script=sci_arttext\&pid=S0325-00752010000200006

Benincasa, M. \& Rezende, M. M. (2006). Tristeza e suicídio entre adolescentes: fatores de risco e proteção. Boletim de Psicologia, 55(124), 93-110. Recuperado de http://pepsic.bvsalud.org/scielo.php?script=sci_arttext\&pi$\mathrm{d}=\mathrm{S} 0006-59432006000100007$

Bloc, L. \& Moreira,V. (2013). Sintoma e fenômeno na psicopatologia fenomenológica deArthurTatossian. Revista Latinoamericana de Psicopatologia Fundamental, 16(1), 28-41. https://doi.org/10.1590/S1415-47142013000100003

Borges, V., Werlang, B., \& Copatti, M. (2008). Ideação suicida em adolescentes de 13 a 17 anos. Barbarói, $28,109-123$. https://doi.org/10.17058/barbaroi.v0i0.192

Bós, A. M. G. \& Bós, A. J. G. (2005). Fatores determinantes e consequências econômicas da depressão entre os idosos no Brasil. Revista Brasileira de Ciências do Envelhecimento Humano, 2(2), 36-46. https://doi.org/10.5335/rbceh.2012.34

Bossarte, R. M. \& Swahn, M. H. (2011). The associations between early alcohol use and suicide attempts among adolescents with a history of major depression. Addictive Behaviors, 36(5), 532-535. https://doi.org/10.1016/j.addbeh.2010.12.031

Bouma, E. M. C., Ormel, J., Verhulst, F. C., \& Oldehinkel, A. J. (2008). Stressful life events and depressive problems in early adolescent boys and girls: the influence of parental depression, temperament and family environment. Journal of Affective Disorders, 105(1-3), 185-193. https://doi.org/10.1016/j.jad.2007.05.007

Calear, A. L., Grifftihs, K. M., \& Christensen, H. (2011). Personal and perceived depression stigma in Australian adolescents: magnitude and predictors. Journal of Affective Disorders, 129(1-3), 104-108. https://doi.org/10.1016/j.jad.2010.08.019

Campos, J. R. (2010). Habilidades sociais de adolescentes com indicadores de depressão: considerando fatores de gênero e socioeconômicos (Dissertação de mestrado). Centro e Educação e Ciências Humanas, Universidade Federal de São Carlos, São Carlos.

Champion, J. E., Jaser, S. S., Reeslund, K. L., Simmons, L., Potts, J. E., Shears, A. R. et al. (2009). Caretaking behaviors by adolescent children of mothers with and without a history of depression. Journal of Family Psychology, 23(2), 156-166. https://doi.org/10.1037/a0014978

Clarke, G., \& Harvey, A. G. (2012). The complex role of sleep in adolescent depression. Child and Adolecent Psychiatric of North America, 21(2), 385-400. https://doi.org/10.1016/j.chc.2012.01.006

Coutinho, M. P. L. (2001). Depressão infantil: uma abordagem psicossocial. João Pessoa, PB: Ed. Universitária.

Coutinho, M. P. L., \& Ramos, N. (2008). Distúrbios psicoafetivos na infância e adolescência: um estudo transcultural. Psico (PUCRS), 39(1), 23-27. Recuperado de http://revistaseletronicas.pucrs.br/fass/ojs/index.php/revistapsico/article/view/1418/2790

Crivelatti, M. M. B., Durman, S., \& Hofstatter, L. M. (2006). Sofrimento psíquico na adolescência. Texto \& Contexto - Enfermagem, 15(n esp), 64-70. https://doi.org/10.1590/S0104-07072006000500007

Cunha, B. F. V., Buzaid, A., Watanabe, C. E., \& Romano, B. W. (2005). Depressão na infância e adolescência: revisão bibliográfica. Revista da Sociedade de Cardiologia do Estado de São Paulo, 15(3 Supl A), 8-16.

Fu-1, L., \& Wang, Y. P. (2008). Comparison of demographic and clinical characteristics between children and adolescents with major depressive disorder. Revista Brasileira de Psiquiatria, 30(2), $124-131$. https://doi.org/10.1590/S1516-44462008000200007

García, J. E. G. A., Montoya, R. Q., Loyo, L. M. S., López, T. M., \& Gaitán, J. I. C. (2011). Consenso cultural sobre el intento de suicidio en adolescentes. Revista Colombiana de Psicología, 20(2), 167-179. Recuperado de http: / / www.redalyc.org/articulo.oa?id=80421265002

Gazalle, F. K., Lima, M. S., Tavares, B. F., \& Hallal, P. C. (2004). Sintomas depressivos e fatores associados em população idosa no Suldo Brasil. RevistadeSaúdePública, 38(3), 365-371.https://doi.org/ 10.1590/S0034-89102004000300005 
Gerhardt, T. E. \& Silveira, D. T. (2009). Métodos de pesquisa. Porto Alegre, RS: Editora UFRGS.

Gladstone, T. R. G, Beardslee, W. R., \& O'Connor, E. E. (2011). The prevention of adolescent depression. Psychiatric Clinics of North America, 34(1), 35-52. https:// doi.org/10.1016/j.psc.2010.11.015

Hale, W. W., Van DerValk, I., Engels, R., \& Meeus, W. (2005). Does perceived parental rejection make adolescents sad and mad? The association of perceived parental rejection with adolescent depression and aggression. Journal of Adolescent Health, 36(6), 466-474. https://doi.org/10.1016/j.jadohealth.2004.04.007

Herman, K. C.; Ostrander, R., \& Tucker, C. M. (2007). Do family environments and negative cognitions of adolescents with depressive symptoms vary by ethnic group? Journal of Family Psychology, 21(2), 325-330. https://doi. org/10.1037/0893-3200.21.2.325

Higgins, J. P. T., \& Green, S. (Eds.). (2011). Cochrane handbook for systematic reviews of interventions: version 5.1.0. London: The Cochrane Collaboration.

Horwitz, A. G., Hill, R. M., \& King, C. A. (2011). Specific coping behaviors in relation to adolescent depression and suicidal ideation. Journal of Adolescence, 34(5), 1077-1085. https://doi.org/10.1016/j.adolescence.2010.10.004

Houaiss. (2009). Dicionário eletrônico Houaiss da língua portuguesa. Rio de Janeiro, RJ: Objetiva.

Jatobá, J. D. V. N., \& Bastos, O. (2007). Depressão e ansiedade em adolescentes de escolas públicas e privadas. Jornal Brasileiro de Psiquiatria, 56(3), 171-179. https://doi.org/10.1590/S0047-20852007000300003

Kubik, M. Y., Lytle, L. A., Birnbaum, A. S., Murray, D. M., \& Perry, C. L. (2003). Prevalence and correlates of depressive symptoms in young adolescents. American Journal of Health Behavior, 27(5), 546-553. https:// doi.org/10.5993/AJHB.27.5.6

Lima, D. (2004). Depressão e doença bipolar na infância e adolescência. Jornal de Pediatria, 80(2 suppl 0), 11-20. https://doi.org/10.1590/S0021-75572004000300003

McCauley, E., Katon, W., Russo, J., Richardson, L., \& Lozano, P. (2007). Impact of anxiety and depression on functional impairment in adolescents with asthma. General Hospital Psychiatry, 29(3), 214-222. https://doi.org/10.1016/j.genhosppsych.2007.02.003

Martins, F. (1999). O que é pathos? Revista Latinoamericana da Psicopatologia Fundamental, 2(4),62-80. Recuperado de http://www.scielo.br/pdf/rlpf/v2n4/1415-4714-rlpf-2-4-0062.pdf

Máximo, G. C. (2010). Aspectos sociodemográficos da depressão e utilização de serviços de saúde no Brasil (Tese de doutorado). Faculdade de Ciências Econõmicas, Universidade Federal de Minas Gerais, Belo Horizonte.

Melo,A.K.S.,\&Moreira,V.(2008).Fenomenologiadaqueixadepressivaemadolescentes:umestudo crítico-cultural.Aletheia, (27), 51-64. Recuperado de http://pepsic.bvsalud.org/scielo.php?script=sci_arttext\&pid=S1413-03942008000100005

Monteiro, K. C. C., \& Lage, A. M. V. (2007). A depressão na adolescência. Psicologia em Estudo, 12(2), 257-265. https://doi.org/10.1590/S1413-73722007000200006

Moreira, V. (2009). Clínica humanista-fenomenológica: estudos em psicoterapia e psicopatologia crítica. São Paulo, SP: Annablume.

Moreira, V. (2007). Critical phenomenology of depression in Brazil, Chile and the United States. Latin American Journal of Fundamental Psychopathology On Line, 4(2), 193-218. Recuperado de http://pepsic.bvsalud.org/ scielo.php?script=sci_arttext\&pid=S1677-03582007000200007

Moreira, V., \& Sloan, T. (2002). Personalidade, ideologia e psicopatologia critica. São Paulo, SP: Escuta.

Morris, M. C., Ciesla, J. A., \& Garber, J. (2010). A prospective study of stress autonomy versus stress sensitization in adolescents at varied risk for depression. Journal of Abnormal Psychology, 119(2), 341-354. https://doi.org/10.1037/a0019036

Muñoz, S. I. S., Takayanagui A. M. M., Santos, C. B., \& Sanchez-Sweatman, O. (2002). Revisão sistemática de literatura e metanálise: noções básicas sobre seu desenho, interpretação e aplicação na área da saúde. In: Anais do $8^{o}$ Simpósio Brasileiro de Comunicação em Enfermagem-SIBRACEN, Ribeirão Preto, São Paulo. Recuperado de http://www.proceedings.scielo.br/scielo.php?pid=MSC0000000052002000200010\&script=sci_arttext

Organização Mundial da Saúde - OMS (1993). Classificação dos transtornos mentais e de comportamento - CID-10. Porto Alegre, RS: Artes Médicas. 
Palos, P., Ocampo, D., Casarín, A., Ochoa, B., \& Rivera, R. (2012). Práticas parentales y sintomatologia depressiva en adolescentes. Salud Mental, 35(1), 29-36.

Pawlby, S., Hay, D. F., Sharp, D., Waters, C. S., \& O’Keane, V. (2009). Antenatal depression predicts depression in adolescent offspring: Prospective longitudinal community-based study. Journal of Affective Disorders, 113(3), 236-243. https://doi.org/10.1016/j.jad.2008.05.018

Pelkonen, M., Marttunen, M., Kaprio, J., Huurre, T., \& Aro, H. (2008). Adolescent risk factors for episodic and persistent depression in adulthood: a 16-year prospective follow-up study of adolescents. Journal of Affective Disorders. 106(1-2), 123-131. https://doi.org/10.1016/j.jad.2007.06.001

Poletto,M.,Koller, S.H., \&Dell'Agilo, D.D. (2009).Eventosestressores emcriançaseadolescentes em situação devulnerabilidadesocialdePortoAlegre. CiênciaeSaúdeColetiva, 14(2), 455-466.https://doi.org/10.1590/S1413-81232009000200014

Reeb, B. T., \& Conger, K. J. (2009). The unique effect of paternal depressive symptoms on adolescent functioning: associations with gender and father-adolescent relationship closeness. Journal of Family Psychology, 23(5), 758-761. https://doi.org/10.1037/a0016354

Rocha, T. H. R., Ribeiro, J. E. C., Pereira, G. A., Aveiro, C. C., \& Silva, L. C. A. (2006). Sintomas depressivos em adolescentes de um colégio particular. Psico USF, 11(1), 95-102. https://doi.org/10.1590/S1413-82712006000100011

Rodríguez de Cossío, A., \& Granada Jiménez, O. (2007). Transtornos depressivos em la infancia y adolescencia. Revista Clínica de Medicina de Familiar, 1(6), 270-276.

Rother, E. T. (2007). Systematic literature review x narrative review. Acta Paulista de Enfermagem, 20(2), 5-6. https://doi.org/10.1590/S0103-21002007000200001

Sampaio, R. F., \& Mancini, M. C. (2007). Estudos de revisão sistemática: um guia para síntese criteriosa da evidência científica. Revista Brasileira de Fisioterapia, 11(1), 83-89. https:// doi.org/10.1590/S1413-35552007000100013

Santana, P. R. (2008). Suporte familiar, estilos parentais e sintomatologia depressiva: um estudo correlacional (Dissertação de mestrado). Instituto de Psicologia, Universidade São Francisco, Itatiba.

Santiago, A., \& Holanda, A. F. (2013). Fenomenologia da depressão: uma análise da produção acadêmica brasileira. Revista da Abordagem Gestáltica, 19(1), 38-50. Recuperado de http://pepsic.bvsalud.org/scielo.php?script=sci_ arttext\&pid=S1809-68672013000100006

Santos, S. A., Lovisi, G., Legay, L., \& Abelha, L. (2009). Prevalência de transtornos mentais nas tentativas de suicídio em um hospital de emergência no Rio de Janeiro, Brasil. Cadernos de Saúde Pública, 25(9), 2064-2074. https://doi.org/10.1590/S0102-311X2009000900020

Schneider, A. C. N., \& Ramires, V. R. R. (2007). Vínculo parental e rede de apoio social: relação com a sintomatologia depressiva na adolescência. Aletheia, (26), 95-108. Recuperado de http://pepsic.bvsalud.org/scielo.php?scrip$\mathrm{t}=\mathrm{sci}$ abstract\&pid=S1413-03942007000200009\&lng=pt\&nrm=iso

Silva Junior, E. A. (2010). Comorbidades psiquiátricas associadas com transtornos de ansiedade em uma amostra de crianças e adolescentes (Dissertação de mestrado). Faculdade de Medicina.,Universidade Federal do Rio Grande do Sul, Porto Alegre.

Souza, L. D. M., Silva, R. S., Godoy, R. V., Cruzeiro, A. L. S., Faria, A. D., Pinheiro, R. T. et al (2008). Sintomatologia depressiva em adolescentes iniciais: estudo de base populacional. Jornal Brasileira de Psiquiatria, 57(4), 261-266. https://doi.org/10.1590/S0047-20852008000400006

Stein, G. L., Curry, J. F., Hersh, J., Breland-Noble, A., March, J., Silva, S. G., et al (2010). Ethnic differences among adolescents beginning treatment for depression. Cultural Diversity and Ethnic Minority Psychology, 16(2), 152-158. https://doi.org/10.1037/a0018666

Stice, E., Ragan, J., \& Randall, P. (2004). Prospective relations between social support and depression: differential direction of effects for parent and peer support? Journal of Abnormal Psychology, 113(1), 155-159. https://doi.org/10.1037/0021-843X.113.1.155

Suplicy, A. M. (1993). Depressão na infância e adolescência. Revista Neuropsiquiatria da Infância eAdolescência, 1(2), 31-34.

Teixeira, P. S., Stefanini, M. C. B., Martins, R. A., \& Cruz, L. A. N. (2011). Desenvolvimento cognitivo e sintomas depressivos em adolescentes que fazem uso de bebidas alcoólicas. Revista Eletrônica Saúde Mental Álcool e Drogas, 7(1), 3-9. https://doi.org/10.11606/issn.1806-6976.v7ilp03-09 
Toros, F, Bilgin, N. G., Bugdayci, R., Sasmaz T., Kurt, O., \& Camdeviren, H. (2004). Prevalence of depression as measured by the CBDI in a predominantly adolescent school population in Turkey. European Psychiatry, 19(5), 264-271. https://doi.org/10.1016/j.eurpsy.2004.04.020

Wagner, M. F., Silva, J. G., Zanettelo, L. B., \& Oliveira, M. S. (2010). O uso da maconha associado ao déficit de habilidades sociais em adolescentes. SMAD. Revista Electrónica Salud Mental, Alcohol y Drogas, 6(2), 255-273. Recuperado de http://pepsic.bvsalud.org/scielo.php?script=sci_arttext\&pid=S1806-69762010000200003

Waiselfisz, J. J. (2012). Mapa da violência 2012: crianças e adolescentes do Brasil. Rio de Janeiro, RJ: Centro Brasileiro de Estudos Latino-Americanos.

Werlang, B. S. G., Borges, V. R., \& Fensterseifer, L. (2005). Fatores de risco ou proteção para a presença de ideação suicida na adolescência. Revista Interamicana de Psicologia, 39(2), 259-266. Recuperado de http://www.psicorip. org/Resumos/PerP/RIP/RIP036a0/RIP03929.pdf

Wisdom,J.P.,\&Agnor,C.(2007).Familyheritageanddepressionguides:Familyandpeerviewsinfluenceadolescentattitudes about depression. Journal of Adolescence, 30(3), 333-346. https://doi.org/10.1016/j.adolescence.2006.04.001

World Health Organization - WHO (2014) Health for the world's adolescents: a second chance in the second decade. Geneva: World Health Organization.

Zinn-Souza, L. C., Nagai, R., Teixeira, L. R., Latorre, M. R. D. O., Roberts, R., Cooper, S. P. et al (2008). Factors associated with depression symptoms in high school students in São Paulo, Brazil. Revista de Saúde Pública, 42(1), 34-40. https://doi.org/10.1590/S0034-89102008000100005

\section{Anna Karynne Melo}

Doutora em Saúde Coletiva pela Associação de IES Ampla (UFC/UECE/Unifor), Fortaleza - Ceará. Docente da Universidade de Fortaleza, Fortaleza - CE. Brasil.

E-mail: karynnemelo@unifor.br

\section{Adolfo Jesiel Siebra}

Graduado em Psicologia pela Universidade de Fortaleza, Fortaleza - CE. Brasil.

E-mail: jesielsiebra@gmail.com

\section{Virginia Moreira}

Doutora em Psicologia Clínicapela Pontifícia UniversidadeCatólica de São Paulo, São Paulo-SP.Pós-Doutora em Antropologia Médica pela Harvard University, Cambridge . EUA. Docente da Universidade de Fortaleza, Fortaleza-CE. Brasil.

E-mail: virginiamoreira@unifor.br

Endereço para envio de correspondência:

Universidade de Fortaleza - Unifor. Diretoria do Centro de Ciências Humanas, Psicologia.

Av. Washington Soares, 1321, Edson Queiroz. CEP: 60811-341.

Fortaleza - CE. Brasil

Recebido $27 / 12 / 2014$

Aprovado 12/12/2016

Received $12 / 27 / 2014$

Approved 12/12/2016

Recibido 27/12/2014

Aceptado 12/12/2016 
Como citar: Melo, A. K., Siebra, A. J., \& Moreira, V. (2017). Depressão em adolescentes: revisão da literatura e o lugar da pesquisa fenomenológica. Psicologia: Ciência e Profissão, 37(1), 18-34. https://doi.org/10.1590/1982-37030001712014

How to cite: Melo, A. K., Siebra, A. J., \& Moreira, V. (2017). Depression in adolescents: review of the literature and the place of phenomenological research. Psicologia: Ciência e Profissão, 37(1), 18-34. https://doi.org/10.1590/1982-37030001712014

Cómo citar: Melo, A. K., Siebra, A. J., \& Moreira, V. (2017). Depresión en Adolescentes: Revisión de la Literatura y el Lugar de la Investigación Fenomenológica. Psicologia: Ciência e Profissão, 37(1), 18-34. https://doi.org/10.1590/1982-37030001712014 